\title{
Close reading und Prosa
}

\section{Einleitende Überlegungen zu Oswald Egger}

Oswald Egger ist einer der wichtigsten Autoren der deutschsprachigen Gegenwartsliteratur, durchaus auch einer der erfolgreichsten. Sein Werk wurde mittlerweile mit zahlreichen Literaturpreisen ausgezeichnet und in mehrere Sprachen übersetzt, obwohl seine Texte für literarische Avantgarde stehen und als unzugänglich gelten. Nicht wenige der Laudator:innen betonen deren Unlesbarkeit, bezeichnen Eggers Textuniversum als hermetisch und beschreiben ihn als >Worterfinder sSprachmagier. In der akademischen Literaturwissenschaft liegen nur einige wenige Aufsätze vor, die sich der Aufgabe einer intensiven Auseinandersetzung mit Eggers Texten stellen. Die vielleicht ambitionierteste Studie ist ein Aufsatz von Andrea Albrecht. ${ }^{1}$ Sie baut darin eine für die literaturwissenschaftliche Beschäftigung mit Egger kennzeichnende Dichotomie auf: Lesbar werden ihr die Texte (erst und nur) dort, wo sie ihr als Exemplifikationen von allgemeineren Modellen (hier besonders der mathematischen Topologie) gelten, während ihr die konkrete Textbewegung, das Fortschreiten von Wort zu Wort und die jeweils besondere ästhetische Faktur der Werke hermetisch bleibt. Albrecht kommt entsprechend zu dem Schluss, dass der Leser durch Eggers Texte an seine hermeneutischen Grenzen geführt werde und - statt zu verstehen und literaturwissenschaftlich zu interpretieren - eben (nur) diese Grenzen des Verstehens reflektieren könne und solle. Die Behauptung, dass Eggers Texte schlichtweg uninterpretierbar ${ }^{2}$ oder fürs Verstehen nicht gemacht seien, ein Verstehen vielmehr prinzipiell und kalkuliert zurückweisen würden, findet sich dabei in gleich mehreren der bislang vorliegenden Studien. ${ }^{3}$ Entsprechend ist die Forschung zu Egger von einem bemerkenswerten

1 Andrea Albrecht: »Auszug aus selbstverschuldeter Verständlichkeit«. Hermeneutisches und Hermetisches zur Poesie Oswald Eggers. In: Scientia Poetica. Jahrbuch für Geschichte der Literatur und Wissenschaften. Bd. 21 (2017), 210-235.

2 Eleonore De Felip: Inwendige Landschaften oder Die leeren Räume der Sprache. Peter Waterhouse' "Spaziergang als Himmelskunst « und Oswald Eggers »Im Anger des Achilles«. Möglichkeiten und Grenzen einer wissenschaftlichen Lektüre. In: Studia austriaca 21 (2013), 51-80, hier $52 \mathrm{f}$.

3 Christina Weiss: »Nicht gemacht für lineares Lesen. Über Oswald Egger«. In: Sinn und Form 59.2 (2007), 277-281, hier 281.

\footnotetext{
Martin Endres, Leipzig

Ralf Simon, Basel
}

https://doi.org/10.1515/9783110690286-001 
Widerspruch gekennzeichnet: Auf der einen Seite gesteht sie den Texten eine große Faszinationskraft zu, die eine intensive Beschäftigung mit ihnen motiviert, zugleich aber herrscht die communis opinio, dass das, was dort zu lesen ist, sich dem Verstehen und der Interpretation entzieht. Werden also permanent lediglich Wortabfolgen gelesen, die offenkundig auf keinen Sinn hin synthetisiert werden, während gleichzeitig eine mittlerweile doch recht andauernde Beschäftigung mit diesen Texten stattfindet? Und welchen Begriff von ıästhetischer Erfahrung`setzt man an, wenn der Text - vermeintlich - keinen wie auch immer gearteten Nachvollzug zulässt?

Charakteristisch für die literaturwissenschaftliche Reaktion auf Eggers Texte ist neben dieser Absage ans hermeneutische Geschäft der Hinweis auf Verfahren eines rein spielerischen Experimentierens, aus dem Wortpermutationen erwachsen und Neologismen hervorgehen würden. So schreibt Björn Vedder in seinem KLG-Artikel:

Solch klangspielerische Kombinatorik zeigt sich auch in der Lust am Neologismus, wie etwa das erste Gedicht im Zyklus »Hänggärten« zeigt (im Band »Prosa, Proserpina, Prosa«): »Früh - nicht für mich - umbern und umenden ihre Nachtschattentage. / Malven, die Spiegelmelde emporpochen Nußwolken Bergnarden. / Melissen grüßen dich, Nepeta, Nesselsalbei munden bald ums Jahr. ${ }^{4}$

Aber geht es hier tatsächlich nur um eine Lust am Sprachspiel, um eine rein selbstbezügliche Kombinatorik der Sprache? Dominiert die Konnotation über die denotativen Werte? Recherchiert man die Wörter der Textstelle aus Eggers Prosa, Proserpina, Prosa, die Vedder zitiert, dann ergibt sich ein ganz anderes Bild - und es zeigt sich eine ganz andere Poetik.

So kann das Wort umbern als eine Verbbildung gelesen werden, abgeleitet von >Umber`, einem im Mittelmeer heimischen Speisefisch, der trommelnde Laute hervorbringt. Hier wird aus dem Gegenstand (Substantiv) eine Tätigkeit (Verb), die zusammen mit der Vorstellung der Nacht eine Unterwasserbewegung, eventuell eine Traumwirklichkeit (Früh - nicht für mich) evoziert, welche in die Zeit (Tage) fällt, in denen Nachtschattengewächse ihre Blüten und Beeren hervorbringen. Diese wiederum sollen Kopfschmerzen erzeugen (so die Auskunft in Adelungs Wörterbuch), gemäß Otto Brunfels im Contrafayt Kreüterbuch von 1534 sogar »wider die schäde die die hexen den leüten zufügen« wirken (S. xcvi). So bildet der erste Vers oder die erste Zeile die Evokation einer nächtlichen Aktivität, die wie unter der Wasseroberfläche, vielleicht also traumhaft in der Frühe, fast kaum vom Wahrnehmenden verantwortet (nicht für mich), dem Einfluss einer Pflanzengattung unterliegt.

4 Björn Vedder: Oswald Egger. In: Kritisches Lexikon zur deutschsprachigen Gegenwartsliteratur (KLG): http://www.munzinger.de/document/16000000774 [07.04.2021]. 
Die zweite Sequenz nimmt das Bild des unter dem Wasser pochenden Fisches auf, seine Laute steigen jetzt nach oben, es etabliert sich die Szene einer Bergwiese (Bergnarde), mit Kriechgewächsen (Malven) und krautigen Strauchpflanzen (Melden). Das Wort Nußwolke bereitet Schwierigkeiten, es ist der Name für einen Nusskuchen, der eine leicht nach oben gewölbte Form hat und gerne mit Puderzucker bestreut serviert wird. Man kann entsprechende Gesteinsformen, mit Morgentau überzogen, assoziieren, wenn man im Vorstellungsbild der Bergwiese bleibt. Gleichwohl, es bleibt bei diesem Wort eine Irritation, die man aufrechterhalten sollte, denn eine tiefergehende Lektüre der Hänggärten wird nachweisen können, dass hier keine Naturlyrik mit intakt evozierten Naturbildern vorliegt, sondern eher ein Surrealismus, der aus der Kombination heterogen bleibender Gegenstandsbereiche resultiert (sofern die Lektüre die Anschauungskorrelate aktiviert). Die krautigen Pflanzen behaupten sich (Melissen, Nepeta, Salbei), aus allen lassen sich Wirkstoffe gewinnen, die Heilkräfte haben und medizinisch genutzt werden; in älteren Texten finden sich entsprechend Querverbindungen $\mathrm{zu}$ Magie und abergläubischen Praktiken. Das Verb munden verweist auf eine Konsumation dieser mitunter drogenhaften Wirkstoffe, was sich mit der Traumatmosphäre des ersten Verses oder des ersten Satzes verbinden lässt.

So könnte sich eine Interpretation gestalten. Aber es lässt sich ebenso eine zweite Deutungslinie ziehen, die ausgehend von umbern eine andere Richtung, und damit einen anderen Sinn verfolgt. Denn umbern kann auch von lat. umber (Schatten) abgeleitet werden, wodurch es als ıschattenhaft ‘ sowie sumber-farbig`, serdfarben lesbar wird. In dieser Deutung wird mit umbern ein Weg zu den Nachtschatten gebaut, die Erdtöne leiten zur Erde (der Rede) und der Farbton zum akustischen Ton und damit zu einer Charakterisierung eines besonderen poetischen Sprechens. So führt diese Interpretation von der Traum- und Halbschlafszene der ersten Lektüre weg und bereitet andere Topographien, die vielleicht durch das Verb umenden eine Umzirkelung des Gebiets der Hänggärten andeuten, sodass ein Beet - ein Gebiet des poetischen Sprechens, das Beet als Alphabet - entsteht, das nun ausbuchstabiert wird.

Ganz gleich, welche Interpretation man verfolgt: Die geduldige Recherche zeigt, dass dieser Passage keine >Worterfindung` zu Grunde liegt, und wohl auch keine Kombinatorik, die primär aus klangspielerischen Zusammenhängen entsteht. Entgegen der Arbitrarität, die in den Begriffen `Spiel`, `Experiment und >Lust am Klang، impliziert ist, entstehen doch sehr konkrete Schreibverfahren sowie relativ stabile Szenographien, die nun - da es schon zu Beginn zwei sind mit Gründen bedacht sein wollen. Sichtbar wird diese Textur, wenn man sich auf grundlegendes philologisches Handwerkszeug besinnt, d. h. sich die Mühe macht, den Wortsemantiken gewissenhaft nachzugehen und die >Wörtlichkeit 
des Textes ernst zu nehmen, statt auf der Oberfläche ihrer lautlichen Eigenschaften entlangzugleiten.

Die Initiative für den vorliegenden Band entspringt der Lektüreerfahrung, dass Eggers Texte dort, wo man sie sozusagen `wortwörtlich nimmt und auf Verbindungen, Verstrebungen und Textlogiken achtet, sehr wohl lesbar und präzise zu interpretieren sind. Solange man Egger als sprachspielenden Hermetiker oder als experimentierenden Kombinatoriker verstehen möchte, wird man sich zu einer solchen Interpretation, wie wir sie eben skizziert haben, nicht aufgefordert sehen. Stimmt wiederum die Vermutung, dass Eggers Textgestaltung immerzu auch semantisch motiviert ist, dann tritt der Rätselcharakter, der seine Texte umgibt, nur umso stärker hervor - zugleich aber erwächst daraus eine nicht mehr zu leugnende Aufgabe für die Literaturwissenschaft. Denn zur bislang konstatierten inventorischen Energie der sprachlichen Permutationen käme hinzu, dass dabei immer auch etwas gedacht und gesagt worden ist, das Deutung nicht nur ermöglicht, sondern nach dieser verlangt.

Dieses Lesbarmachen besteht unseres Erachtens in einem Verfahren, das präzise am Text der Schrittweise des close reading folgt. ${ }^{5}$ Es ist um strenge Philologie zu tun, also um das Einfachste und zugleich Schwierigste. Einfach scheint dieses Tun auf den ersten Blick nicht zuletzt deswegen, weil die einschlägigen Wörterbücher und Lexika, die für die Analyse und Recherche der Wortbedeutungen essentiell sind, mittlerweile größtenteils digitalisiert vorliegen. In Folge aber stellt sich die ungleich komplexere Aufgabe, mit diesem `Befund interpretatorisch umzugehen und sich den Problemen des Verstehens zu konfrontieren.

Ist man nun der Ansicht, Literaturwissenschaft müsse ihrem Namen gerecht werden, also Wissenschaft von der Buchstäblichkeit der poetischen Texte sein, dann stellt sich trotz aller >Hilfsmittel schnell heraus, dass dieses Projekt nicht oder nur kaum durchführbar ist. Es gibt zwar Arbeiten, die sich als Monographien einem einzigen Gedicht widmen; dieser Textumgang markiert dabei aber schon fast die Grenze des so Leistbaren. Ein konsequent durchgeführtes close reading erfordert, rein quantitativ gesprochen, gegenüber dem Ausgangstext einen exponentiellen Diskursumfang. Somit ist das Vorhaben, Die ganze

5 Close reading und das deutsche Pendant immanente Interpretation sind keine unbelasteten Begriffe, sie hängen in der Fachgeschichte durchaus mit sehr konservativen Habitus zusammen. Alternativen wie etwa Radikalphilologie, textnahe Interpretation oder strenge Philologie (ggf. sogar als philological turn) existieren, haben sich aber nicht durchsetzen können. Unser Votum hängt durchaus nicht am Begriff des close reading, benutzt ihn aber nicht zuletzt auch deshalb, um eine denkbar scharfe Abgrenzung gegen das distant reading mitzuführen. Letztlich wird es darum zu tun sein, dem Begriff des genauen Lesens durch die exegetische Praxis sein Ethos zuzutragen. 
Zeit, Eggers opus magnum, textnah zu interpretieren, angesichts der über 700 Seiten eine Unmöglichkeit. Gleichwohl bleibt die ethisch zu nennende Aufgabe - im Sinne einer Ethik der Lektüre -, so zu lesen, dass nicht einfach nur Schlüsselstellen einer Exegese unterzogen werden, um dann durch kulturwissenschaftliche Narrative den Raum zwischen ihnen flugs zu verfugen. In diesem `Zwischen nämlich steht zunächst der ungelesen gebliebene Text. Und die Erfahrung zeigt, dass dessen Lektüre schnell dazu führt, dass die vermeintlichen Schlüsselstellen aufhören, solche zu sein.

Wie also ist zu lesen? Paul de Man nannte es nicht umsonst eine verzweifelte Angelegenheit. - Die in diesem Band vorgelegten Studien unternehmen dennoch den Versuch. Sie entrichten den dafür notwendigen Preis, indem sie sich auf relativ kurze Textsequenzen und Passagen beschränken, diese aber so zu lesen versuchen, dass zumindest der Intention nach nichts übersprungen wird.

Interessanterweise fordern die Texte Eggers ein solches Vorgehen geradezu heraus. Denn es ist schwer, in ihnen überhaupt 'Schlüsselstellen ` auszumachen: Die Unterscheidungen von Haupt- und Nebenhandlung, von Thema und Rhema, von Exposition und Durchführung treffen auf sie nicht zu. Dies wiederum hat unseres Erachtens nicht zuletzt auch mit einer besonderen Verschränkung von Lyrik und Prosa zu tun.

Folgt man den Überlegungen von Agamben, dann basiert Lyrik insofern auf der Versförmigkeit, als der Vers ein gerichtetes Formtelos kennt, auf das sich der nächste zugeordnete Vers hinsichtlich der Form korrespondierend ins Verhältnis setzt. Prosa hingegen, so Agambens spekulative Volte, hat infolge des Fehlens eines Formbegriffs die Möglichkeit, jedes sprachliche Momentum sofort in Selbstreferenz zu versetzen, es zu wenden und zu drehen, es auf sich selbst zurückzuführen. Der Vers kann zwar ebenfalls über sein Formtelos hinausgehend weitere Selbstbezüglichkeiten realisieren, diese dürfen jedoch die Versförmigkeit als solche nicht in Frage stellen. Der Prosatext hingegen kann schon im ersten Wort dessen integre Gestalt außer Kraft setzen, es in sich zerspalten, etwa so, wie Arno Schmidts Verschreibkunst (Vers-Reib-Kunst) eine Etym-Schreibweise etabliert. Wo Form ihre Gestalt wahren muss und also eine Stoppregel hinsichtlich ihrer Komplexität mit sich führt, kann die Selbstreferenz der Prosa beliebige Rekursionen freisetzen und geradezu Komplexität als wesentliche ästhetische Verfahrensweise affirmieren.

Es zeigt sich, Agamben fortführend, dass Form und Selbstreferenz zwei durchaus unterschiedliche Prinzipien sind, infolge derer Prosa die Möglichkeit gewinnt, gegenüber der Lyrik die komplexere, dichtere Textur auszubilden. Diese Aussage steht im evidenten Widerspruch zu der langen Tradition der Poetik, nach der die Lyrik das Paradigma poetischer Verdichtung gewesen ist. Die überraschende Volte, bestimmte Prosatexte quasi als Konkurrenz zu diesem Paradigma aufzubie- 
ten, geht einher mit der ebenfalls verblüffenden Feststellung, dass es sich dabei nicht selten um sehr umfangreiche Texte handelt. Zettel's Traum, Abend mit Goldrand, Finnegans Wake, die Geschichtklitterung: Im Gegensatz zum Gedicht geht hier intensivste Verdichtung nach innen mit einer Bewegung der textuellen Expansion einher. Dieser Prozess ist durchaus rätselhaft, er wird wohl nur innerhalb einer Theorie poetischer Selbstreferentialität einigermaßen zu erhellen sein.

Oswald Eggers Texte arbeiten in intensivster Weise in diesem Spannungsfeld von Lyrik und Prosa. Oben wurde ganz bewusst offen gelassen, ob die Textzeile in den Hänggärten-Texten Vers oder Prosasatz ist. Kalkuliert gehen die Sätze immer genau bis zum rechten Textrand, sodass der für die Verszäsur typische Weißraum nicht entsteht, gleichwohl aber der Textblock die eigentümliche visuelle Gestalt eines Gedichtkörpers annimmt. Die Hänggärten versetzen die Frage, ob der Prosasatz ein Vers oder eine Zeile ist, in den Zustand einer Oszillation, die auf die Lektüre zurückschlägt. Eng mit dieser Frage verbunden ist die Bedeutung des Verbums. Denn der Prosasatz erzeugt seinen propositionalen Gehalt durch die Relation von Subjekt und Prädikat, welche durch das Verb gebildet wird. Wenn im oben diskutierten Textauszug die Verben umbern (sofern es eines ist), umenden, emporpochen, grüßen, munden sind, dann fallen gewisse phonetische Rekurrenzen zwischen umbern, umenden und munden auf, sodann eine Verbableitung aus einem Substantiv, welche in pochen semantisch verdeutlicht wird. Vereinzelt steht das Verb grüßen, welches als aus einer Prosopopöie resultierende Anrede ans Ich gedeutet werden kann. Das wäre ein lyrisches Verfahren, während die Sätze ihren konstativen Aussagegestus beibehalten, also prosaähnlich sind, aber darin zugleich das Lyrische dadurch übersteigen, dass sie, indem sie primär Selbstreferenzen etablieren, nicht mehr die Formklammer des Verses benutzen. Den Hänggärten ist nicht beizukommen, wenn man sie als Ich-Rede in Versen (Lamping) verstehen wollte. Sie sind eher polyphone Prosarede, die die Versform als weiteren Modus von Selbstreferenz in sich einkopiert.

Egger arbeitet solche Szenen der >distinkten Oszillation` (Jean-Luc Nancy) von Prosa und Lyrik insistent durch, in Nichts, das ist, in Prosa, Proserpina, Prosa und auch in Die ganze Zeit, deren Textblöcke als Variantenreihe der Lyrik/Prosa-Unterscheidung lesbar sind. Aus dieser Grundanlage resultiert die Eigentümlichkeit, es mit so intensiven Selbstbezüglichkeiten zu tun zu haben, dass diese selbst schon wieder eine Art von Fläche bilden, die sich ihrerseits zu sich selbst verhält. Deshalb gibt es in dieser Textualität tendenziell keine Schlüsselstellen - und deshalb ist ein close reading einiger Passagen die ihr angemessene literaturwissenschaftliche Herangehensweise.

Die Beiträge des vorliegenden Bandes widmen sich dieser Arbeit, ein genaues Lesen zu versuchen und dabei zugleich Erkenntnisse zur Lyrik/Prosa-Unterscheidung zu gewinnen. Folgerichtig erscheint er in der Reihe Theorie der Prosa. 
ELEONORE DE FELIP widmet sich Eggers 2017 erschienenem Val di Non durch die Lektüre der Paratexte (Klappentext, `Schlusswort`) und der ersten, unpaginiert gebliebenen Seiten. Die Ortskenntnis des Nonsbergs (ital. Val di Non) führt schon auf den ersten Seiten zu aufschlussreichen Differenzbestimmungen zwischen poetischer Realität und realer Referenz. Denn Eggers Buch arbeitet mit den Mythen des Nonsbergs, mit seiner Real- und Naturgeschichte, mit seiner Flora und Fauna, mit der Arbeits- und Lebenswelt der dort beheimateten Menschen: Es ist die Vielzahl von Referenzen, die eine Referenznahme verhindert. Statt die poetische Welt in die fruchtlose Unterscheidung zur Realität zu stellen, weist De Felip auf das Gegenteil hin. Es geht um ein Übermaß von Bezugnahmen, um Mehrfachbestimmungen durch übereinander gelagerte Zeiten und sich ergänzende Analogiebildungen. So wird das Val di Non zur sSumme vieler denkbarer Täler` (so De Felip) oder zur Summe vieler Denkbarkeiten eben dieses Tals. Je genauer De Felip den Worten nachspürt - meist auch ihren dialektalen Herkünften desto präziser zeigt sich Eggers Textur als Versenkung in genau diejenige Sprachebene, die so intensiv mit dem Realen verflochten ist, dass sie nicht Sprache über etwas ist, sondern aus den Tätigkeiten, den Mythen und den Gegebenheiten hervorgeht. So wird der Text von De Felip in mehrfacher Sichtweise rekonstruiert: als konkreteste Sprachlandschaft, als Hesiod'sche Mythogenese dieses Tals, verstanden als Weltmodell, als szenisches Bilderbuch einer zwischen Prosa und Lyrik changierenden Bewegung und schließlich als Figurenlehre der coincidentia oppositorum. Diesem komplexen Aufriss gelten die genauen Lektüren der ersten (unpaginierten) Seiten des Buches: präzisestes close reading, im Kontakt zu den vielen aufgerufenen Registern, von Descartes über Hesiod und Jean Paul bis zum Sagengut Tirols und diversen weiteren Schichten, sprachlichen wie geologischen.

Der Beitrag von TILL DEMBECK wirft eine methodologische Frage auf, die im Kontext des vorliegenden Bandes brisant ist. Wenn Eggers Texte von einem grundsätzlichen Isomorphiegesetz aller Ebenen zueinander - im Sinne der Riemann'schen Fläche - geprägt sind, kollabiert dann nicht die Unterscheidung von close versus distant reading? Man kann, wie in diesem Band geschehen, einen einzigen Satz von Egger lesen und in ihm alles eingefaltet finden; man kann aber auch die Logik der Ausfaltung schlichtweg nachzählen. Dies tut Dembeck. Er zählt die Vierzeiler aus nihilum album (2007) und ergänzt dies durch die Zählung von bestimmten Worten hinsichtlich ihrer Verteilung im Text. Dabei wird eine gewisse Beliebigkeit zwischen Semantik und Wortverteilung deutlich, etwa wenn hinsichtlich der Jahreszeiten der Winter bevorzugt wird, selbst wenn die Texte nicht-winterliche Szenarien aufwerfen. Eine solche Zufälligkeit resultiert, so Dembeck, aus dem permutativen Grundzug von Eggers Schreiben, einer extremen Parataxe zwischen den Vierzeilern, die dadurch zueinander in eine Äquidistanz treten, in der immer Bezüge vorhanden sind, ohne dass sie priorisierbar 
wären. So sind semantische Felder benennbar, bleiben aber, durch gleichzeitig kontrainduzierende Nachbarschaften auch undeutlich. Man kann mithin nie sagen, ob die Texte ıgelten`, verworfen oder schlicht geworfen - umhergeworfen, durcheinandergeworfen - werden. Dem close reading wird somit die Vorsetzung einer intensiven punktuellen Textkohärenz zumindest teilweise entzogen, während die Kohärenzverfahren auf der Ebene der Textgruppierung und der Übergänge zwischen den Texten $\mathrm{zu}$ finden sind, also bei einer Dimension, die sich einem distant reading erschließt. Folglich: Bei Egger konvergieren close und distant reading. Dembeck stößt auf die für Egger zentrale Figur, Textnähe und Textferne als notwendige Wechselbewegung denken zu müssen. Dembecks Studie, die ihren Grundgedanken in einer Folge von Neuansätzen durchführt, kommt zu weiteren wichtigen Beobachtungen. Hinsichtlich des Vokabulars von Egger und seiner Wortbildungsverfahren lässt sich feststellen, dass der Wortschatz eine Dingwelt adressiert, die zumindest vorindustriell ist und in starker Bezogenheit auf das >Ich evoziert wird. - Ein letzter Gedanke des facettenreichen Aufsatzes sei hervorgehoben: Die Vierzeiler erinnern an die deutsche Liedtradition. Aber Dembeck beobachtet ein Gegeneinander von Klanglichkeit und Ikonizität, begleitet von einer eigentümlichen Situationsabstraktheit der Vierzeiler. Dies, flankiert durch die kaum mögliche Memorierbarkeit der Kurztexte, unterwandert ihren Liedcharakter und führt überraschend zum Begriff der Prosa als formtranszendente Hybridisierung der selbstreferentiellen Register des Poetischen.

PETER Gilgens Aufmerksamkeit gilt der Lektüre eines Satzes auf der letzten Seite von Oswald Eggers Val di Non. Dieser Satz, der erste und längste von sieben, die auf zwei kurze, philosophisch grundierte Eingangsfragen zum ontologischen Status von Dichtung Antwort geben, bringt - so die dem Beitrag zugrunde gelegte These - das zur Sichtbarkeit, was Gilgen als `Medium der Literatur ‘ fasst, i. e. den im Normalfall unbeachteten und als gegeben vorausgesetzten historischkontextuellen Bezugsrahmen, in dem die literarischen Formen eingeschrieben sind und in dem sich jedes Werk situiert. Vor dem Hintergrund dieses `Mediums und im Zuge von dessen kritischer Reflexion werden, so Gilgen, literarische Formen erst zu solchen. Die von Egger am Ende des Satzes zitierten Verse des Horaz (in Wielands Übersetzung) enthalten beträchtliche unausgewiesene Abweichungen gegenüber dem Quelltext, auf den er ausgreift und mit dem er arbeitet. Die kalkulierten Anomalien markieren formal und inhaltlich die Grenzen der imitatio als dichterischer Strategie, die im darauf folgenden zweiten Satz, der die ruminatio zum Thema hat, überschritten werden. Das Widerspiel der beiden historisch spezifischen Arten der Textverarbeitung lässt eine Nähe von Eggers Verfahrensweise zur Poetik Petrarcas erkennen. Gilgens Lektüre vermag aufzuzeigen, dass diese und weitere literarische Genealogien, die Egger nicht selten mit Hilfe von versteckten Allusionen und abgewandelten Zitaten konstruiert - etwa die Serie 
von literarisch bedeutungsvollen Tälern am Anfang des ersten Satzes -, die Notwendigkeit bezeugen, poetische Formen auf das hin zu befragen, was Gilgen mit Luhmann als den »Raum sinnvoller kompositorischer Möglichkeiten « fasst, der mit jedem Text aufgerufen und zugleich transformiert wird.

FRANZISKA HuMPHREYS analysiert in ihrem Beitrag eine Passage aus Oswald Eggers Prosa, Proserpina, Prosa mit Blick auf Intertextualität und Mehrsprachigkeit und schlägt vor, die semantischen Verdichtungsprozesse und Verflechtungen, die der Text aufweist, als eine Form poetischer >Ligatur` $\mathrm{zu}$ begreifen. Gegenstand ihrer Untersuchung ist hauptsächlich der Abschnitt »Im Anger des Achilles«, für den Humphreys ausgehend von der Verschränkung vom englischen sanger` (Zorn) und dem deutschen >Anger` (Acker) für einen literarischen Raum argumentiert, der sich `zwischen den Sprachen aufspannt und eine Trennung der semantischen und der lautlich-sinnlichen Dimension des Textes nicht mehr zulässt. Eggers zum Teil mikrologische Operationen am Sprachmaterial sind, so zeigt Humphreys, keine formalistischen Spielereien, sondern eine präzise poetische Arbeit am und mit dem Quelltext - eine hochkomplexe Anverwandlung, durch die bereits Gesagtes (übertragen und verformt) neu interpretiert und in anderem >Areal rekontextualisiert wird. Der Gedanke der >Ligatur` als reflexive Engführung verschiedener Texte und Sprechweisen gewinnt schließlich auch für die Frage nach der >Prosa v von Eggers Dichtung Bedeutung: Mit ihm kann sich der Spannung zwischen den Textblöcken in der oberen Seitenhälfte von Prosa, Proserpina, Prosa, die eine bewusste Überblendung von Zeilen- und Versrede kennzeichnet, und dem Sprachfluss am Fuß der Seiten genähert werden. `Ligatur` kann aber auch, so Humphreys, das Verhältnis von Text und Bild, von Schreiben und Zeichnen im ästhetisch-aisthetischen Buchraum beschreibbar machen, das spätere Werke wie Die ganze Zeit oder Val di Non prägt.

Der Beitrag von RALPH KAUFMANN kann als `Grundlagenforschung`zum Werk von Oswald Egger gelten. Denn obwohl sich Kaufmann hauptsächlich mit Harlekinsmäntel \& andere Bewandtnisse. $A-Z$ beschäftigt, setzen seine Ausführungen vor jedem Einzelwerk von Egger an. Mit der Zergliederung und Rekombination (`Teilen` und `Fügen`) des Sprachmaterials, der Philosophie von Leibniz und Cusanus sowie der mathematischen Topologie werden zentrale Momente von Eggers Poetik diskutiert, die entscheidende Zugänge zu seinem Gesamtwerk eröffnen. Es ist dabei vor allem die Perspektive auf Eggers Texte, durch die der Beitrag einen so propädeutischen Wert erhält: Kaufmann liest Egger nicht nur als literaturwissenschaftlich und philosophisch gebildeter Leser, sondern vor allem als Mathematiker. So zeigt er, dass Leibniz' Monadologie, Eggers poetologische Reflexionen als Teil seiner immanenten Poetik, seine Bezugnahme auf dichtungstheoretische Quellen und die besondere Textstruktur und Logizität seiner Werke ihren Verknüpfungspunkt in der mathematischen Flächen- und Raumtheorie der Topologie finden. 
Entsprechend gewinnen die auch in Eggers Texten thematisierten und zugleich realisierten poetischen Verfahrensweisen wie das >Verkleben` und `Schneiden`,

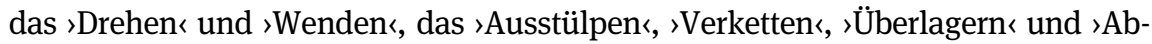
bilden ‘ mit der Topologie nicht einfach eine zusätzliche Bedeutungsebene hinzu vielmehr kommt ihnen in der Terminologie der Mathematik gefaßt erst die Geltung zu, die Eggers poetisches Denken angemessen nachvollziehbar macht. Wie grundlegend sich die Wechselwirkung von Dichtung und Mathematik vor allem in Harlekinsmäntel \& andere Bewandtnisse. $A-Z$ die Topologie ausgestaltet und wie vielfältig sich in und durch Wortverbindungen poetisch-mathematische Figuren und Muster sowie literarische Räume aufbauen, die mit den Zeichnungen kommunizieren und hier ihr anschauliches Komplement erhalten, bezeugt Kaufmanns präzise Lektüre einzelner Abschnitte im zweiten Teil seiner Studie.

CLAUDIA KELLERS Lektüreprotokoll basiert auf einem Zufallsfund. Im nicht publizierten Schlussbericht eines Stipendienjahres der Villa Massimo findet sich ein sehr eigentümlicher Rechenschaftsbericht von Oswald Egger. Keller nähert sich dem Text in einer methodischen Geste, die ausdrücklich die Assoziationen und passiven Synthesen des Leseaktes mitzuformulieren versucht. Entsprechend ist ihr Text in der Ichform geschrieben. Er stellt die Beobachtungen in den konjekturalen Raum von Vermutungen, unterfüttert diese aber durch Bezugnahmen auf Lexika, die Auskunft über Eggers Wortschatz geben. So entsteht ein tastender Gestus, der ein Geflecht von Wahrscheinlichkeiten ausbreitet. Keller entwickelt dabei eine konkrete Phänomenologie der Lektüre, die vom Schauen auf die Textdarbietung langsam in ein Lesen übergeht, welches die seltsame Beobachtung macht, dass sich die Rezeptionszustände mit dem decken, was im Text Eggers artikuliert wird. Diese Konvergenz von Textprozess und Rezeptionsprozess ruft stark induzierte Einverständnisse hervor, Keller spricht $u$. a. von einem Schneetreiben des Textes oder von seiner Kreisform, der sich der Lektüreakt kaum entziehen kann. Dieses hermeneutische Eingenommensein endet abrupt, als in Eggers Text der Name von Siegfried Passarge auftaucht, eines bedeutenden Geographen und Paläontologen, der im Dritten Reich Mitglied der NSDAP war und der in seinen Schriften eine rassistische und antisemitische Kolonialpolitik vertritt. Egger, der im Rechenschaftsbericht seines Stipendienjahres seine Lektüren dokumentiert, tut dies in der Weise, in der alle seine Texte gearbeitet sind: Der Fremdtext geht in seinen eigenen über, Grenzen werden nicht markiert, es entstehen intertextuelle Kontinua. Ist dies angesichts eines faschistischen Fremdautors ein Problem? Kellers weiterhin sehr vorsichtige und tastende Lektürebewegung kann zumindest feststellen: Eggers Textualität wehrt sich gegen diesen Fremdtext nicht, sein Schreiben ist auf dieser Ebene apolitisch, ein gewisser Hang zu einer vormodernen Dingwelt auf der Gegenstandsebene seiner Texte schafft eine Atmosphäre, 
die einigen Zügen bei Passarge entgegenkommt. Keller verhandelt also »die Grenzen einer rein immanenten Auseinandersetzung mit literarischen Texten ebenso [...] wie die Bedeutung der Kontexte« bei der Textlogik, die für sie einen verführerischen Sog bereitet, welcher nun, durch den unverdaulichen Fremdtext, beschädigt worden ist. - Claudia Kellers Studie ist freilich selbst auch verführerisch, gerne folgt man zunächst einer so genau beschriebenen Erotik der Lektüre. Aber man kann Egger philosophischer lesen, sogar mathematisch, mit starken Argumenten für komplexe Selbstreferenzen. Eine Eggerlektüre, die sich bereit zeigt, in Eggers Immanenz mitzuschwimmen (u. a.: »Klang-Bad«) und die im ১Schnaüggen eine durchaus genießerische Haltung zur phoné einnimmt, begibt sich selbst in eine schwierige Situation, wenn plötzlich Textinhalte auftauchen, die eine Ethik der Lektüre und den Begriff des Politischen auf den Plan rufen. Ein anderer Textbegriff und eine andere Rezeptionsbewegung würden mit solchen Inhalten auch anders umgehen können. Gleichwohl: Kellers mit äußerster Dezenz vorgehender Aufsatz macht auf ein Problem, vielleicht auf ein Befremden aufmerksam, welches weiterwirkt.

JÖRG KREIENBROCK nimmt sich eines zentralen Aspekts von Oswald Eggers Dichtung an: der Darstellung von Landschaft. An -broich (2003) entwickelt Kreienbrock, dass Eggers `Sprachlandschaft « in diesem Text als eine grundlegend >gebrochene und `brüchige` begriffen werden kann. Der >Bruch ‘ der poetischen Rede ist dabei einer, der über die Referenz auf die geomorphologische Bodenbeschaffenheit bestimmter Gegenden (`Broich`als `Sumpfgebiet`) und der Region von Städten und Dörfern mit der Endung >-broich ein poetisches Grundmotiv Eggers artikuliert. Zum einen lässt sich, so Kreienbrock, in der Bezugnahme auf die >Ruderalflora` von mit >Broich <enannten Landstrichen der Kölner Bucht eine in der Sprache sich ausgestaltende Rekultivierung und Renaturierung beobachten, die sich als poetische Rekombination und Neuverbindung von Wörtern aus der Botanik vollzieht. Die Logik dieser Trans- und Deformation ist - wie im Untertitel von -broich angegebenen - die der Homotopie, d. h. der kontinuierlichen Veränderung von Abbildungen im Sinne der mathematischen Topologie, dergemäß das poetische Sprechen nicht im Schöpfen von Neologismen, sondern in der Umgestaltung von Bestehendem erfolgt. Den >Bruch der Rede erkennt Kreienbrock zum anderen in der >gerasterten` Darstellung von Landschaft, die eine ständige Oszillation zwischen Figur und Grund, zwischen der Ordnung des `Sprachgitters` und der Ordnung des Dargestellten in Gang setzt. Die poetischen Rasterungen erweisen sich dabei als durchaus mehrfach überlagert, Moiré-Effekte sowie dynamisch-komplexe Muster ausbildend. Das Gitter der Rede ist bei Egger zugleich Sieb und Reuse, um Wörter aus alltäglichen Zusammenhängen herauszulösen, abzuschöpfen und schließlich in neue Beziehungen zu stellen. 
ANDREA SAKOPARNIG und MARTIN ENDRES widmen sich in einem äußerst präzisen close reading einem paradigmatischen Satz aus Oswald Eggers Nichts, das ist. Sie verfolgen die These, dass der Satz als Aufkündigung eines uns vertrauten Textverständnisses, vom Text als - wie sie es nennen - >monadologischem Ordnungsraum $\mathrm{zu}$ verstehen ist sowie als Exemplifikation eines neuen Textverständnisses, vom Text als einem komplex mannigfaltigen Raum. Dazu zeichnen Sakoparnig und Endres detailliert die Auflösung von rhetorischen (wie >Wort für Wort ) und logischen (Denk-)Figuren (etwa dem aristotelischen sunbewegten Beweger ) nach und zeigen, dass diverse Operationen des (Auseinander-)Dividierens die sprachlichen Elemente aus dem konkreten und sie konkretisierenden Zusammenhang lösen, sie vereinzeln und isolieren. Dies führe zu einer Elementarisierung und (Para-)Metrisierung des Sprachmaterials, die eine reflexive wie konstruktive Bedeutung besitzen. Der analytische Vorgang sei als eine Inventur des sprachlichen und literarischen Materialbestands zu begreifen, die die Elemente jedoch nicht positivistisch sichte, sondern in der ihnen gemeinhin zukommenden Funktion reflektiere. Eine besondere Leistung des Beitrags von Sakoparnig und Endres besteht darin, dass er am Text nachvollziehbar macht, welch konstitutive Rolle das logische und mathematische Denken für Eggers Poetik spielt: Der Ausgriff auf das formalsprachliche Sprechen, den metrisierten Mengenraum und die Riemannsche Fläche sind hier nur einige wenige Beispiele. Sakoparnig und Endres weisen überdies auf, dass die Genese des komplex mannigfaltigen Textraums durch eine Dreh-, Wendungs- und Umkehrungslogik bestimmt ist: So könne nicht nur vom Kippen konkreter und konkretisierter sprachlicher Elemente $\mathrm{zu}$ abstrakten und abstrahierten die Rede sein, die letztlich die Individuation als Division auslege; auch die Assoziation wende sich in die Dissoziation, der Vers werde invers, Invarianten würden variiert, Definites indefinit, Besonderes allgemein, Eines zu Vielem, Logisches zu Analogischem, das Stetige diskret und das Diskrete stetig, der Zusammenhang verkehre sich in den Unzusammenhang u.v.m. All das verdeutliche, dass Egger mit einem sowohl reversiven (vielleicht gar revisionären) als auch invasiven Gestus am Materialbestand arbeitet. Die Lektüre von Sakoparnig und Endres macht diese poetische Arbeit als eine an mathematischen, (topo)logischen sowie handwerklichen Praktiken orientierte erfassbar, die als $\mathrm{Mo}$ delle, Ressourcen und Paradigmen der Textgenese gleichermaßen wirksam werden.

Von einer ganz anderen literaturwissenschaftlichen Geste ist der Text von Thomas Schestag. Es gibt in der Literaturwissenschaft die Schreibweise einer langsamen, in die Wortwörtlichkeit des Materials hinabsteigenden Recherche. Wohl für keinen Autor ist sie angemessener, als für Oswald Egger. Man könnte Schestags Werk - und also auch seinen Aufsatz in diesem Band - als literaturwissenschaftliche Parallelaktion zu Eggers Texten lesen. Schestags Überlegungen heben mit dem Begriff des Exakten an, führen ihn zum kategorialen Denken und 
nutzen eine etymologische Spur zum Verb ágein, um vom zusammentreibenden Haben zu Eggers Herde der Rede (1999) zu gelangen. Dies wird mit weiteren Etymologien verbunden - cogo, cogito, Herde (poímne), Hirt (poimén), Gedicht (poíema) -, sodass ein dichtes Netz von sprachlichen Übergängen und Nachbarschaften entsteht, welches Eggers poetisch-poetologische Nomenklatur abbildet - auf exakte Weise. Egger lesen, heißt also nach Schestag: In Eggers Wortverschaltungen einen Grund lesbar zu machen, der >objektiv` ist, sofern man solche Objektivitäten den etymologischen Quergängen zusprechen möchte.

RALF SIMON beginnt seinen Text recht unvermittelt mit einigen close readings aus Eggers Die ganze Zeit (2010). Die Prosablöcke (Prosagedichte) scheinen, so die an einigen dieser Texte unternommene Gegenprobe, eine intendierte Komplexität zu besitzen. Sie lässt sich als inversive Bewegung bestimmen, in der der ikonische Prozess gegen die Klangtexturen geführt wird. Die Vierzeiler hingegen entspringen, so Simons Beobachtung, als Sprachgebärden einer seriellen Produktion, die eine Nähe zur Permanenz mündlich orientierter Geistesbeschäftigung aufweist. Es handelt sich, in Prosa zurückgeschrieben, um einfache Sätze, die unilinear eine Aussage treffen, während die Prosagedichte schon grammatisch inversive Logiken besitzen. Beide Schreibweisen führen auf Zeitbestimmungen. Den zeittheoretischen Nukleus von Eggers opus magnum erkennt Simon in einer an Husserl angelehnten Zeitphänomenologie, in der aber die stets leerbleibende Gegenwart durch eine Konkretion aufgefüllt wird, die in den Zeitphilosophien stets unthematisiert bleibt. Dies ebnet den Weg zu Eggers Grotesken, die als unfertig gebliebene Zeit bestimmbar werden. Egger arbeitet mit der abendländischen Zeitphilosophie von Augustin bis McTaggart und Husserl, aber die Philosopheme werden jeweils in eine Konkretion überführt, die, durchaus gegen Husserl, nunmehr eine tatsächliche Phänomenologie des inneren Zeitbewusstseins genannt werden kann. So arbeitet Egger das Haus der Memoria (Augustin) ebenso um wie Husserls Protention und Retention, die er als Redeschnüre, wie Fäden im Inneren eines Seils verflochten, denkt. Erneut führt ein Wörtlichnehmen zeittheoretischer Basisannahmen dann zu einer Abfolge grotesk bleibender Unvollständigkeiten, wenn man das jeweilige Prosagedicht als eine konstitutiv unfertige Gestalt solcher Verwicklungen denkt. Simons Überlegungen münden in eine Diskussion der Lyrik/Prosa-Unterscheidung: Paradoxerweise zeigen sich die Vierzeiler als reine Prosa, nämlich als vollkommen geradeausgehendes Sagen, während die Prosablöcke, verstanden als Prosagedichte, mehrfach an komplexe Lyrik - etwa Rilke - erinnern. So wird eine Unterscheidung aufgeworfen, aber nur, um sofort die eine Bestimmung auf die Seite der anderen einzukopieren. Es entsteht erneut eine Oszillation, als Darstellung nicht darstellbarer Zeit.

ALISHA STÖCKLIN geht der Frage nach dem >Denken des Gedichts `Gedicht des Denkens`(Diskrete Stetigkeit, Vorsatzblatt) sowie ihrer Stellung 
zueinander nach. Eggers Zirkularitäten machen den Text als Bild und die Bilder darin als Text lesbar, semantische und schriftbildliche Dimension erzeugen wechselseitig Figur-Grund-Relationen. Entsprechend thematisiert Stöcklin Eggers Selbstverspiegelungen infolge einer Verdichtung der Analogien, die über mehrstufige Abbildverhältnisse läuft. Dabei führt der Aufsatz zunehmend zu einer Analyse von Eggers Integration abstrakter naturwissenschaftlicher Theoreme, die im Medium der Poesie nicht nur eine neue Anschaulichkeit erzeugt, sondern im Rückstoß auch für die Poesie explanatorische Kräfte freisetzt. Gerade durch diese naturwissenschaftlichen Theoreme, die durch Stöcklin intensiv rekonstruiert werden, wächst Eggers Texten eine antihermeneutische Dimension zu, welche wiederum eine Methodenreflexion auf der Seite der Literaturwissenschaft in Gang setzt. Ein konzeptuelles Denken, das aus einem mimetischen Nachvollzug der Prozessualität dieses Materials hervorgeht, muss, so Stöcklins bildtheoretisch untermauerte These, zwischen Begriff und Bild mäandern, immerzu unterscheidend zwischen einem diskursiven Fortschreiten und seiner Negation, woraus sich in zweiter, metaschematischer Reflexion auf die Textbewegung das Denken des Denkens als Flucht- und Ausgangspunkt des Denkens des Gedichts herausstellt. Die sstetige Deformation erscheint zum Schluss als Prinzip, das sowohl das Sprachmaterial wie auch die gedankliche Aneignung desselben durchwirkt. Was das Gedicht ist, kann nie auf den Begriff gebracht werden, obgleich es unablässig danach verlangt. Die fortwährende Suche nach der Einheit seines Begriffs ist aber gerade sein genuiner Geburtsort: Es keimt im >Dreh der Rede` und des Gedankens und erwächst dem ewigen analogischen Prozedieren, >Wort für Wort`.

Überblickt man die Aufsätze in diesem Band, die eine erstaunliche Vielfalt hinsichtlich ihrer Methoden und Schreibweisen aufweisen, dann zeigt sich doch eine intensive Gemeinsamkeit. Fast jeder Text reflektiert über die Frage des eigenen literaturwissenschaftlichen Procederes. Schon der im Vorfeld dieser Publikation ergangene Austausch zwischen den Beiträger:innen und den beiden Herausgebern hat gezeigt, dass das Schreiben über Oswald Egger sehr spezifische Herausforderungen stellt. Vorderhand haben die Beiträger:innen ein Finalproblem: Ihre Texte werden mitunter sehr lang; manche waren durchaus noch länger, als sie in der Druckfassung erscheinen. Das Schreiben über Eggers Dichtung versetzt die Literaturwissenschaft offenkundig in die Situation, sowohl den eigenen Methodenbegriff hinterfragen zu müssen als auch die eigene Schreibbewegung durchaus nicht mehr als konzise Durchführung einer vorab gefassten These zu erfahren. Die Autor:innen haben darauf unterschiedlich reagiert: Till Dembeck etwa durch eine überraschende Konvergenz von close reading und distant reading, Peter Gilgen durch eine Problematisierung einer $\mathrm{zu}$ eng gefassten Vorstellung stextimmanenter Interpretation, Claudia Keller durch eine in der Ichform belassene `Lektüregeschichte`, Thomas Schestag durch eine Art mimetischer Parallelaktion, 
Andrea Sakoparnig und Martin Endres durch die Lektüre eines einzigen Satzes und der von diesem und seiner poetischen Logizität eingeforderten Revision der Methode >close reading`. Offenkundig führt Oswald Egger die sich ihm widmende Literaturwissenschaft ihrerseits in eine Situation enger Selbstbezüglichkeit und Selbstreflexion, in der die Frage nach dem Schreiben auch auf dieser Seite, die sich normalerweise das Schreiben nur zum Objekt der eigenen Tätigkeit macht, aufgeworfen wird. Die Unterscheidung zwischen Prosa und Lyrik, die in fast allen Texten meist gegen Ende hin explizit wird, aber untergründig die Diskurse steuert, sucht auch die literaturwissenschaftliche Schreibbewegung heim. Sie kann nicht mehr nur aus einer prosaischen Prosa bestehen, sondern hat Verfahrensweisen in sich aufzunehmen, durch die sich die Differenz zwischen Literatur und Literaturwissenschaft zwar nicht (wie Paul de Man sie einmal fasst) als >Lug und Trug ^ offenbart, die aber bezeugt, dass beide Seiten in einer kritischen und dynamischen Aushandlung stehen.

Die beiden Herausgeber danken den Beiträgerinnen und Beiträgern zu diesem Band für ihre engagierten Texte. Zur Fertigstellung haben durch intensive und kundige Korrekturen und Lektorate beigetragen: Nicolas Fink, Emmanuel Heman und Maximilian Huschke. Oswald Egger hat uns großzügig die Vorlagen für die Abbildungen in diesem Band zur Verfügung gestellt. Auch ihm sei herzlich gedankt. 
